\title{
Complications of spinal manipulation
}

Assendelft WJJ, Bouter LM, Knipschild PG: Complications of spinal manipulation: A comprehensive review of the literature. J Fam Pract 1996;42:475-480.

Background: Spinal manipulative therapy (SMT) is a frequently applied therapy for back and neck pain. Serious complications of SMT are presented primarily in case reports. Many patients seen by physicians also seek care from non medically qualified therapists applying manipulative techniques. Therefore, background information on the risks of SMT is essential for physicians. Methods: Relevant case reports, surveys, and review articles were identified using a comprehensive search of online and bibliographical databases. For everey case a record was made of the first author, publication year, country, age and sex of the patient, background of the manipulator, preexisting conditions, type of complication, and course of the complication. Based on case reports and surveys, an estimation was made of the risk for the most frequently reported complications: verte-brobasilar accidents (VBAs) and cauda equina syndrome (CES).

Results: 295 complications of spinal manipulations were derived from the literature: 165 VBAs; 61 cases with disc herniation or progression to CES; 13 cerebral complications other than VBAs; and 56 other types of complications. The average age of patients with VBA was 38 years. Vertebrobasilar accidents occur mainly after a cervical manipulation with a rotatory component. Estimates of VBA range from 1 per 20,000 patients to 1 per 1 million cervical manipulations. The incidence of CES is estimated to be less than 1 per 1 million treatments. Conclusions: It is difficult to estimate the incidence of SMT complications, as they are probably underreported in the literature. Most non-VBA complications can be prevented by excluding patients with contraindications for SMT. Patients

who develop complications such as CES should be treated as soon as possible. VBAs, however, are difficult to prevent and treat. Referral for SMT should not be made to practitioners applying rotatory cervical manipulation. Information about the risk of VBA should be included in an informed consent procedure for cervical manipulation with thrust techniques.

Commentary - P.D. Aker, Toronto, Canada

Considerable debate (and many strong opinions) has been raised about the risks associated with manipulative treatment. Historically, spinal manipulation is among the most ancient forms of medicine, having been practiced for almost 5,000 years. In non-westernized cultures manipulation is still widely practiced by traditional healers. In North America and in many European and Scandinavian countries, spinal manipulation is classified as a complementary or alternative form of health care. With recent review evidence supporting the use of manual treatments for various low back and neck disorders [1-3], manipulation is now again being considered by some as a mainstream approach for these conditions [4, 5]. Given this new-found acceptance, Drs. Assendelft, Bouter and Knipschild have provided the health-care community with a timely review of its potential harmful effects.

This article was unfortunately reduced such that one cannot truly evaluate the quality of the review methods, for a detailed report one may contact Dr. Assendelft. Familiarity with the methods used in this and other reviews from the same group, however, allows me to reassure the 
reader of the robustness of this review. My main criticism of this review does not lie in their methods, but rather in the quality of information from which they draw their conclusions. Regardless of how many case reports or surveys were found, the biases inherent in these designs will likely produce spurious results. Without prospective studies, recommendations on health policy or practice guidelines arrived at from these data may be misleading. For

\section{KÄRGER}

(C) 1997 S. Karger GmbH, Freiburg Fax (0761) 4520714 http://www.karger.ch

example, 'referral should not be made to practitioners applying rotatory cervical manipulation' is likely an overstatement, since less than half of the cases reported in the world literature actually describe the manipulative procedure [6]. In spite of this deficiency, Assendelft et al.'s review offers an informative overview of the most common complications from manipulation. Serious complications arising from spinal manipulation appear to be extremely rare. For vertebrobasilar accidents resulting from cervical spine manipulation, the best estimate appears to be in the range of 0.5 to 1 per one million manipulations [2]. For cauda equina syndrome arising from lumbar spine manipulation, Shekelle et al. [1] have estimated the risk to be about 1 in one hundred million manipulations. However rare, these events may be life-threatening or cause permanent impairment and therefore need to be considered seriously. Patients should be wary of receiving manual treatments from unqualified persons. While it may be that basic manipulative techniques can be taught in a series of seminars, probably the most important consideration is to know when NOT to manipulate.

Health care practitioners performing manual treatments should be trained in and proficient with diagnostic skills. Recognition of the early signs and symptoms of stroke and cauda equina syndrome are essential. Although some practitioners use various forms of provocation or positioning to attempt to identify patients at risk, there are as yet no clinical tests that can accurately predict which patients may have an adverse event. Other skills must be incorporated before attempting these treatments - 'above all, do no harm'. For patients undergoing cervical spine manipulation, a thorough medical history including specific questions related to previous stroke or other cardiovascular event, coagulopathy or collagen vascular disorder is essential. Dizziness or unsteadiness must be investigated carefully before attempting neck manipulation. Practitioners must also be able to recognize medications (in particular anticoagulants) which may predispose patients to these events. Careful evaluation is necessary before initiating lumbar manipulation in a patient with progressive neurological deficit. Signs and symptoms of cauda equina syndrome are a surgical emergency. Obtaining a patient's informed consent prior to initiating treatment is strongly recommended. In conclusion, while I think the article is informative and a critical read for practitioners using manual treatments, I'm not convinced it gives the non-manipulator an unbiased estimate of the risks associated with this procedure compared to others. I was disappointed that Assendelft et al. did not commit to an opinion on whether spinal manipulation is safe. Sometimes perspective can help with these decisions: compare the risk of spinal manipulation to that of taking an aspirin (or other NSAID), where the risk of a serious gastrointestinal event (such as bleeding, perforation, or other event resulting in hos-pitalization or death) is estimated at about 1 in one thousand. Given these figures, perhaps it is best to let the reader decide which treatment is safest!

References

Shekelle PG, Adams AH, Chassin MR, Hurwitz EL, Brook RH: Spinal manipulation for lowback pain. Ann Intern Med 1992;117:590-598. 
Hurwitz EL, Aker PD, Adams AH, Meeker WE, Shekelle PG: Manipulation and mobilization of the cervical spine: A systematic review of the literature. Spine 1996;21:1746-1760.

Aker PD, Gross AR, Goldsmith CH, Peloso P: Conservative management of mechanical neck pain: A systematic review and meta-analysis. BMJ 1996;313:1291-1296.

Bigos SJ (chair), Bowyer O, Braen G, et al: Acute low back problems in adults: Clinical practice guideline No. 14. AHCPR Publication No. 95-0642. Rockville, MD: Agency for Health Care Policy and Research, Public Health Service, US Department Health and Human Services.

December 1994.

Waddell G, Feder G, et al: Clinical guidelines for the management of acute low back pain: Low back pain evidence review. London, Royal College of General Practitioners, 1996.

Terrett AGJ: Vertebrobasilar stroke following manipulation. West Des Moines, IA: National Chiropractic Mutual Insurance Company, 1996:34.

Contact address: Dr. Peter Aker, MSc, DC, FCCS(C), Associate Professor, Division of Graduate Studies and Research, Canadian Memorial Chiropractic College, 1900 Bayview Avenue, Toronto ON, Canada M4G 3E6

Kommentar - G. Marx, Hittenkirchen

Es ist den Autoren dieser Arbeit sehr hoch anzurechnen, dass sie sich mit den Komplikationen der Wirbelsäulenmanipula-tion in ihrer Arbeit beschäftigen. Dieses Thema ist anerkannt schwierig, da die bisher zur Verfügung stehenden Daten zu ungenau sind und erheblich divergieren. Im deutschsprachigen Raum gibt es auf Universitätsstandard keinerlei Forschung zu dieser Thematik. Leider geben die Autoren nicht an, warum sie sich mit dieser Literaturübersicht beschäftigt haben, was also die Konsequenz ihrer mühsamen Arbeit sein soil. Ebensowenig geben sie an, für welche medizi-nischen Kreise diese Literaturübersicht bestimmt ist. Die Leser von «Family Practice» werden durch eine Reihe nicht erläuter-ter und nicht definierter Fachausdrücke sicherlich überfordert. Den Autoren sei aber nochmals ausdrücklich gedankt für ihre Arbeit, denn jeder Beitrag, der geeignet ist, die Zwischenfall-quote bei Wirbelsäulenmanipulationen zu senken, ist nützlich und willkommen.

Folgende Anmerkungen und Ergänzungen halte ich persönlich für wichtig:

Diese ausführliche Literaturübersicht bezieht sich fast aus-schliesslich auf Angaben aus den USA und ist mit den ent-sprechenden Verhältnissen in Europa nur sehr einge-schränkt vergleichbar. Die Verhältnisse wechseln bereits erheblich von Staat zu Staat in den USA; das soziale Umfeld der Patienten ist ebenso wenig vergleichbar wie die Bedin-gungen im Gesundheitssystem.

Chiropraktiker in den USA leisten zur Thematik dieser Arbeit in den USA einen auch zahlenmässig sehr bedeutsamen und wichtigen Beitrag, in Europa ist ihr Beitrag von untergeordneter Bedeutung.

126

Forsch Komplementärmed 1997;4:125-132

Journal Club

«Low back pain and neck pain» werden als wesentliche Indikation für die Anwendung der Wirbelsäulenmanipulation angegeben. Wenn eine so ungenaue Krankheitsbezeichnung für eine solch gezielte Therapie angegeben wird, wird das Ergebnis ebenso schlecht sein, als wenn man die Krankheitsbezeichnung «Bauchschmerzen» als Indikation für eine Magenoperation angibt. «Low back pain and neck pain» muss über eine genaue Anamnese, eine exakte Untersuchung mit Feststellung der gestörten Funktion (hypomobil/hyper-mobil), der Segmenthöhe, der 
gestörten Bewegungsrichtung, der Reversibilität, der neurophysiologischen Störungen im zugehörigen Metamer (z.B. Bindegewebsreaktion, Organ-beteiligung, muskuläre Reaktion, Funktion der Zirkulation) und der Stadienangabe akut/chronisch einer genauen Diagnose zugeführt werden. Darüber ist der Patient in verständlichen Worten aufzuklären und nachfolgend der Therapieplan zu entwickeln. Besonders im Bereich der Halswirbelsäule und des Schädels sind häufíg ergänzende Zusatzuntersuchungen aus anderen Fachgebieten nötig. Röntgenaufnahmen der Halswirbelsäule in mindestens zwei Standardebenen sind in standardisierter Aufnahmetechnik und guter Bildqualität obligatorisch, sofern eine Manipulation vorgesehen ist bzw. der klinische Untersuchungsbefund eine solche Röntgenuntersuchung nötig macht, auch wenn keine Manipulation vorgesehen ist.

Nur bei Einhalten dieser Kriterien wird man eine genaue Diagnose erhalten und darauf eine entsprechend gezielte (Manipulations)Therapie aufbauen können. Zusatztherapien aus der Physikalischen Medizin, der Neuraltherapie, der Akupunktur, der Phytotherapie, der Homöopathie und die Anwendung von Entspannungsverfahren werden die Thera-pieergebnisse erheblich und, was sehr wesentlich ist, auch dauerhaft verbessern. Eine wesentliche Ergebnisverbesse-rung auf Dauer lässt sich auch durch eine gezielte Physio-therapie im Sinne der Rehabilitation erreichen, wobei der Patient aktiv zunehmend Aufgaben zu seiner dauerhaften Gesundung zu übernehmen hat. «Activities of daily living» gehören dazu ebenso wie die Diätetik im Hippokratischen Sinne. Mit einer derartigen weitgefächerten, dem Krank-heitsbild und seinem Stadienwechsel angepassten Therapie werden Blockierungsrezidive seltener und damit auch die Manipulation seltener, was einer Risikominderung entspricht.

Aus dem oben Gesagten ergibt sich, dass es nicht sinnvoll ist, die Effektivität der chiropraktischen Wirbelsäulenmanipulation gegenüber der Physiotherapie in der Behandlung des «low back pain» zu prüfen; beide Verfahren ergänzen sich und gehören zusammen. Die detaillierten Diagnosen, die sich unter dem sehr unscharfen Begriff «low back pain» verbergen, lassen ohnehin den hier angestellten Vergleich beider Therapiemethoden in ihrer Effektivität ins Leere laufen. Als ganz einfaches Beispiel: Ein hypermobiles Wir-belsegment lumbale 4-5 macht ebenso tiefsitzende Rücken-schmerzen wie ein blockiertes, also hypomobiles Segment. Im ersteren Fall ist die Manipulation überhaupt nicht indiziert

und wird allenfalls eine Zunahme der Beschwerde $\prod$ bewir-ken. Im zweiten Fall kann eine frühzeitig angew $7 / 8$ ndte Manipulation die Physiotherapie überflüssig mache $\prod$ oder erheblich verkürzen.

Bezüglich der Problematik der arteriellen Versorguๆg des Schädels ist die einseitige vollständige Unterbrechu $\prod g<$ jer Passage der A.vertebralis folgenlos, sofern die andere $\prod$ zum Kopf führenden Gefässe in ihrer Passage frei sind. Eine vollständige Unterbrechung der vorher freien Passage einer A. vertebralis durch extreme Bewegungen der Halswirbelsäule ist bisher nicht beschrieben worden, allenfalls eine Passageminderung. Auch diese Passageminderung wird in Untersuchungen aus jüngster Zeit angezweifelt.

Die Tabelle 1 machte leider keine Angaben

über die Zahl der Manipulationen pro Jahr pro Therapeut,

über die Zahl der Manipulationen insgesamt, zu denen die 295 Zwischenfälle gehören, über den Anteil der ärztlichen Tätigkeit, der aus Wirbelsäulenmanipulation besteht. Diese Angaben sind aber als Mindestforderung zu betrach-ten, um das Risiko der Wirbelsäulenmanipulation annä-hernd beurteilen zu können. Hier fehlen also entscheidend wichtige Daten. 
Wünschenswert wären Angaben zu wesentlichen Vorer-krankungen, exakte Diagnosen und genaue Beschreibung der angewandten Manipulationstechnik. Die Angabe eines mittleren Alters (38 Jahre) bei 165 Komplikationen ist viel zu ungenau, zumal wieder die Angaben über die Gesamt-zahl der Manipulationen fehlen, auf die sich die 165 Komplikationen beziehen. Sehr wichtig wären Angaben zu Alter und Geschlecht der erwähnten 29 Todesfälle und die Obduktionsergebnisse, sofern verfügbar.

Für den Satz «lt is very likely, that the number of complications is actually higher...» wird weder eine logische Be-gründung noch gar ein Beweis erbracht.

Es fehlen leider jegliche Vergleichszahlen über die Häufig-keit des Auftretens von Gefässkomplikationen im Kopf-Hals-Bereich nach Land, Alter, Geschlecht, Lebensgewohnheiten (Raucher/Ernährung). Interessant natürlich wären Angaben darüber, wie oft die Obduktion von To-desfällen durch Wirbelsäulenmanipulation ernsthafte vor-bestehende Gefässläsionen aufgedeckt hat, die durch die Manipulation dekompensiert sind.

Das Nutzen-Risiko-Verhältnis der Wirbelsäulenmanipulation ist dann sehr gut, wenn die Punkte 3 und 4 exakt ein-gehalten, Kontraindikationen ausgeschlossen werden sowie eine adäquat gute Manipulationstechnik angewandt wird. Für akute und chronische hypomobile segmentale reversible Funktionsstörungen ist die schmerzfreie gezielte Manipulation der Wirbelsäule die Methode der Wahl. Ergänzend sind häufíg Zusatztherapien angezeigt.

Die Discusläsion ist keine Indikation für die Manipulation, da es sich dabei nicht um eine reversible, hypomobile segmentale Funktionsstörung handelt.

Journal Club

Forsch Kompleraentärmed 1997;4:125-132

127

«Cervical manipulation with rotatory component* ist eine sehr ungenaue Angabe: Wieviel Grad Rotation, Seitneigung, Flexion, Extension nimmt dabei die Halswirbelsäule ein? Der Impuls erfolgt mit wieviel Grad in die weitere Rotation, wieviel Traktion? Handelt es sich jeweils um endgra-dige Einstelmngen? Diese Angaben sind unumgänglich wichtig, um Aussagen über das zu erwartende Risiko machen zu können.

$\mathrm{Zu}$ einer wesentlichen Risikominderung ist es nötig, das blockierte Segment in alien drei Raumebenen an seine «Barriere» einzustellen. Dieser Ausdruck ist nicht er-wähnt, ebensowenig werden die sehr risikoarmen Metho-den der Muskelenergietechniken, der postisometrischen Relaxation und der osteopathischen Releasetechniken aufgezählt.

Kontraindikationen werden häufig mit Nichtindikationen verwechselt. Eine akute Fraktur ist eben keine Kontraindi-kation, sie ist überhaupt kein Objekt der Manualtherapie. Bei einer relativen Kontraindikation «age greater than 50» wären 50\% der Patienten von der Manipulationsbehand-lung auszuschliessen. Das ist eine absolut unzulässige, fach-lich nicht gerechtfertigte Aussage. Solche schriftlich festge-legten Aussagen haben eine erhebliche forensische Bedeu-tung und können sich für Patienten und Therapeuten sehr nachteilig auswirken.

Zusammenfassung: Aus dieser Literaturübersicht erhält der Hausarzt keine schlüssige Information über das Risiko, die Häufigkeit und die Art der Komplikationen der Wirbelsäulenmanipulation in Europa. Die aus der Literatur übernomme-nen Daten wurden ungenügend analysiert und sind deshalb zu wenig aussagekräftig für die aufgeworfene Problematik. Die 
Kontraindikationen sind in ihren Aufzählungen einerseits unvollständig, andererseits übervorsichtig. Zur Vermeidung von Komplikationen sind unabdingbar:

Genaue Anamnese, ausführlicher Untersuchungsbefund mit Dokumentation, eventuell

Zusatzuntersuchungen aus ande-ren Fachgebieten, an der Halswirbelsäule obligatorisch die Röntgenaufnahmen.

Entwicklung eines darauf aufgebauten Therapieplanes und die verständliche Informierung des Patienten über die Be-funde und das beabsichtigte therapeutische Vorgehen.

-Verwendung therapeutischer Zusatzverfahren bis hin zur Rehabilitation.

- Verantwortungsbewusstsein des Therapeuten, gute funktio-

nell-anatomische und allgememmedizinische Kenntnisse für

die Differentialdiagnostik, ergänzt durch eine gute Hand-

grifftechnik und immer durch ein gewisses Mass an Respekt

vor seinem Handeln.

So wird er bei ständiger Fortbildung sicher weniger Komplikationen und bessere

Heilungsergebnisse haben als der Thera-peut, der «low back pain and neck pain» ungezielt mit der Wir-belsäulenmanipulation behandelt.

Kontaktadresse: Dr. Gerhard Marx, Facharztfür Orthopädie, Chirotherapie und Sportmedizin, Dozent der Deutschen Gesellschaft für Manuelle Medizin, Hochriesstrasse 6, D-83233

Hittenkirchen, Germany

Adresse für Sonderdrucke: WJJ Assendelft, Institute for Research in Extramural Medicine, Vrije

Univeriteit, van der Boechorststraat 7, NL-1081 BT Amsterdam, the Netherlands

Adverse effects of acupuncture

Norheim AJ, Fønnebø V: Acupuncture adverse effects are more than occasional case reports:

Results from questionnaires among 1,135 randomly selected doctors and 197 acupuncturists. Compl Ther Med 1996;4:8-13.

Objective: To evaluate what kind of adverse effects of acupuncture Norwegian doctors and acupuncturists have encountered and to indicate how often these adverse effects occur. Methods: A questionnaire was sent to 1,466 randomly selected physicians (sample frame Norwegian Medical Association membership file) and all 311 acupuncturists listed in the yellow pages of the Norwegian telephone directory. Physicians/acupuncturists were asked if they have ever seen a patient with acupuncture adverse effects. If the answer was yes, type and frequency of adverse effects should be described. Results: 1,135 physicians (77\%) and 197 acupuncturists (68\%) sent back the questionnaires. 7\% of the responding acupuncturists were physicians, $7 \%$ nurses, and $34 \%$ physiotherapists; $41 \%$ had no health profession education. Adverse effects were reported from $31 \%$ of the acupuncturists and $12 \%$ of the physicians. The most common serious adverse effect was pneumo-thorax, which was observed in 33 patients. Local skin infections $(n=66)$, perichondritis on the auricle $(n=6)$, arthritis and osteomyelitis $(n=4)$ are reported from physicians, but not from the acupuncturists. Fainting during treatment is relatively common in the acupuncturists' practices $(n=140)$, while physicians report more cases of psychiatric problems $(n=20)$ and increased pain after treatment $(n=56)$. The rate of acupuncture adverse effects is estimated at least $0.21 \%$ per year of practice as an acupuncturist.

Conclusions: Acupuncture adverse effects are more than occasional case reports. The results imply more focus on who should practise acupuncture and when to use it. Despite the reported adverse effects, acupuncture can still be presented as a relatively safe therapeutic measure.

Commentary - C.F. Borchgrevink, Oslo 
To choose a specific treatment is always a balanced decision where you weigh advantages (positive effects) of the treatment against the disadvantages (adverse effects). There seems to be a general belief at least in the population that complementary medicine is natural and therefore safe and without side effects.

L28

Forsch Komplementärmed 1997:4:125-132

Journal Club 